\title{
Dynamic resource allocation based on a TCP-MAC cross-layer approach for DVB-RCS satellite networks
}

\author{
Paolo Chini ${ }^{1, \star}$, Giovanni Giambene ${ }^{1, *, \dagger}$, Danilo Bartolini ${ }^{2,}$, Michele Luglio ${ }^{2,}$ \\ and Cesare Roseti ${ }^{2, \|}$ \\ ${ }^{1}$ CNIT-University of Siena, Italy \\ ${ }^{2}$ University of Rome 'Tor Vergata', Italy
}

\begin{abstract}
SUMMARY
Satellite communication networks can provide multimedia broadband services to fixed and mobile users in several scenarios where terrestrial networks are not present or where they need to be complemented. Moreover, satellite links can be useful to bypass crowded terrestrial networks, thus helping in reducing congestion. In such a scenario, characterized by a high propagation delay and a radio channel affected by losses, standard bandwidth allocation schemes prove to be inefficient when Transmission Control Protocol (TCP) is running. Hence, we propose a novel resource allocation scheme based on the cross-layer interaction between TCP and Medium Access Control (MAC) layers. The interest is here in achieving an efficient transfer of files by means of the FTP application protocol. Our scheme permits to avoid network congestion, to reduce the average file transfer time and to achieve a fair sharing of resources among competing flows. The allocation technique has been applied to an Interactive Satellite Network (ISN) based on the DVB-RCS standard with a group of fixed satellite terminals, which communicate with a Network Control Center (NCC) through a geostationary bent-pipe satellite, according to a classical star topology. Results are obtained through a network simulator. Copyright (C) 2006 John Wiley \& Sons, Ltd.
\end{abstract}

Accepted 5 July 2006

KEY WORDS: cross-layer design; satellite networks; DVB-RCS; TCP

\footnotetext{
*Correspondence to: Giovanni Giambene, Dipartimento di Ingegneria dell'Informazione-Università degli Studi di Siena, Via Roma, 56, 53100 Siena, Italy.

†E-mail: giambene@unisi.it

E-mail: chini7@unisi.it

${ }^{\S}$ E-mail: danilo.bartolini@uniroma2.it

๑E-mail: luglio@uniroma2.it

${ }^{\|}$E-mail: cesare.roseti@uniroma2.it

Contract/grant sponsor: European Commission; contract/grant number: 507052
}

Copyright (C) 2006 John Wiley \& Sons, Ltd. 


\section{INTRODUCTION}

Satellite networks have an important role since they can be the unique communication medium in inaccessible regions on the land masses or in the Oceans; moreover, they are the best candidate for a fast provision of broadband communications in very large areas on the Earth. Despite these attracting characteristics, satellite networks pose special design challenges especially due to the high propagation delays, the impact of adverse weather conditions (rain for frequencies beyond $10 \mathrm{GHz}$ ), shadowing in case of mobility, and the management of timevarying traffic loads. Dynamic network management and related control algorithms are therefore necessary for the support of data transmissions with Quality of Service (QoS) guarantees.

Many popular Internet applications, including e-mail, file transfer, remote access and Web browsing, require a reliable delivery of data. End-to-end reliability for Internet traffic is guaranteed by the Transmission Control Protocol (TCP) at the transport layer [1]. TCP is based on a sliding window mechanism; in particular, a congestion window (cwnd) is used to control the injection of traffic in the network [1]. Unfortunately, TCP does not distinguish between segment losses due to network congestion (i.e. buffer overflow) and those caused by the radio link. In the latter case, where losses are independent of network congestion, the drastic reduction of the cwnd value (and hence of the TCP throughput) is inappropriate. Moreover, TCP suffers from the high delays of the satellite scenario since its traffic injection in the network is ACK-based [2].

In this paper, we refer to a satellite network based on the Digital Video Broadcasting-Return Channel via Satellite (DVB-RCS) standard [3,4], where a Network Control Center (NCC), enriched with gateway functionality, manages the allocation of radio resources to terminals for transmission through a Geosynchronous Earth Orbit (GEO) bent-pipe satellite. Such system is here analysed in the presence of traffic generated by file transfers (FTP application). The scope of this paper is to propose a novel Bandwidth on Demand (BoD) scheme, centrally controlled by the NCC, that performs an optimized resource allocation by means of a cross-layer interaction between Medium Access Control (MAC) and TCP. The proposed allocation technique assigns resources to TCP flows according to the terminal requests, encapsulated into the MAC header of data packets. Moreover, this scheme allows the co-existence with other allocation schemes (e.g. the proposed cross-layer BoD scheme is employed for all low-priority TCP-based traffic flows, while other policies are used to manage real-time traffic).

A comprehensive survey on cross-layer methods for BoD is provided in Reference [5]. Moreover, cross-layer issues on DVB-RCS satellite networks have been addressed in References [6-9]. In particular, the authors in Reference [6] study a level 2 queue and propose a BoD scheme to support TCP-based traffic over GEO satellite networks; they use an equation-based algorithm to predict TCP flows. Moreover, BoD mechanisms are also described in Reference [7], considering their impact on the TCP behaviour. In Reference [8], the authors propose a family of BoD criteria and present a new adaptive allocation scheme, able to support TCP-based traffic, which considers the traffic characteristics and the size of the transmitted files. In Reference [9], the authors study different combinations of TCP versions and link layer techniques; the aim is to evaluate the impact at the TCP level due to the interactions of lower layers.

Our BoD technique for TCP-based traffic flows is original with respect to the literature for the following aspects: (i) resource requests are made in advance on the basis of a prediction of the TCP behaviour (based on the TCP status), in order to reduce the access delays of classical BoD 
schemes; (ii) a resource-aware congestion control is operated by the NCC and superimposed to the end-to-end classical congestion algorithm. It is therefore evident that our innovative BoD scheme exploits a twofold cross-layer exchange of control-information between layers 2 and 4 at both user terminals and the NCC [10]; our aim is to prove that the proposed BoD scheme outperforms other BoD schemes of DVB-RCS.

This paper is organized as follows: Section 2 describes the system reference architecture based on the DVB-RCS standard; Sections 3 analyses DVB-S and DVB-RCS air interface resources and how they can be managed; Section 4 describes TCP mechanism; Section 5 introduces crosslayer design issues and contains the description of our TCP-based BoD scheme; Section 6 shows simulation results and, finally, Section 7 provides conclusions.

\section{SYSTEM REFERENCE ARCHITECTURE}

Our reference DVB-RCS architecture has a star topology where user terminals are directly connected to a central hub while they need a double hop to exchange data among each other. The DVB-RCS system envisages a time-division multiple access transmission in the return link (from terminals to the hub) and employs the Digital Video Broadcasting-Satellite (DVB-S) standard for the forward link traffic (from the hub to terminals).

The main macro elements of the network are: the space segment and the ground segment (Figure 1). The former consists of a GEO bent-pipe satellite. The latter is composed of:

- The Network Operation Center (NOC), which includes the hub for network interconnection, the NCC that operates acquisition/synchronization, Radio Resource Management (RRM), alarm management, security management, performance management, billing, and accounting;

- User interactive terminals (hereafter referred to as Return Channel Satellite Terminals, RCSTs), communicating with the hub through a return channel via satellite.

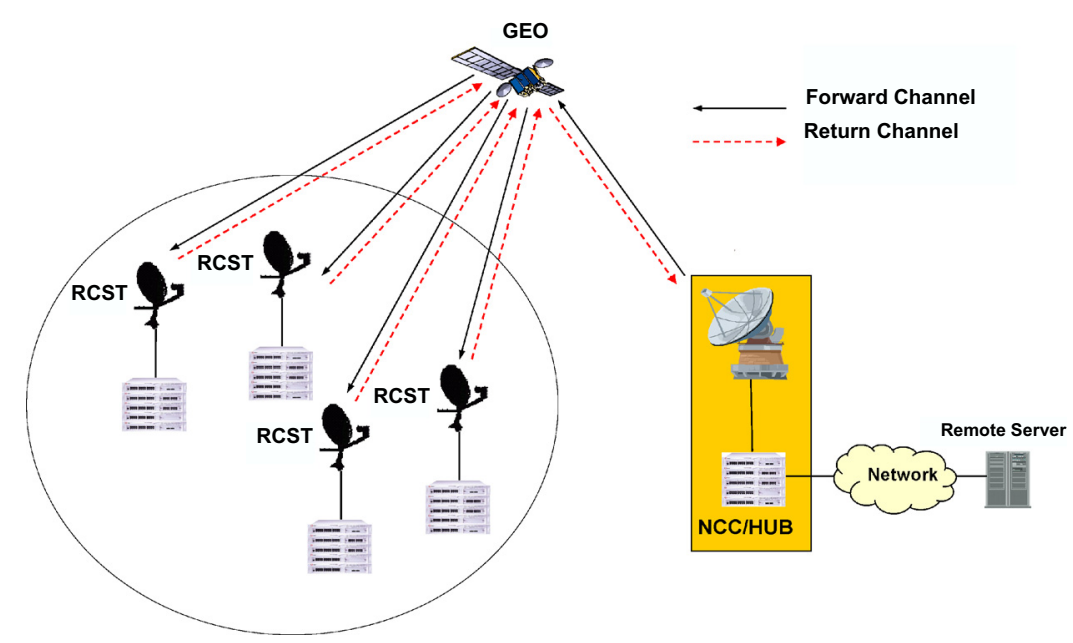

Figure 1. Reference architecture. 
An RCST can even represent the aggregation point of multiple users, but in this paper, without loosing in generality, a single user per RCST will be considered, since this is a simple assumption that permits to evaluate the management of TCP-based traffic flows via satellite. The consideration of more users per RCST is left to a future study.

\section{DVB-RCS AIR INTERFACE AND RESOURCE MANAGEMENT}

DVB-S has been conceived for primary and secondary distribution (Fixed Satellite Service, FSS) and Broadcast Satellite Service (BSS), at present mostly operated in the Ku band $(11 / 14 \mathrm{GHz})$ [11]. This system is designed to provide a direct reception from the satellite (Direct-To-Home, DTH) for both a single user with an integrated receiver-decoder and a collective access. In our work, we have analysed the characteristics of data transmission and resource management in DVB-RCS return link; further details on forward link are out of the scope for this paper.

Referring to the return link, below the transport layer and the IP layer, Multi Protocol Encapsulation (MPE) provides segmentation and reassembly functions for the generation of MPEG2-TS (Transport Stream) packets of fixed length (188 bytes). A TCP header of 20 bytes, an IP header of 20 bytes and an MPE header + CRC trailer of $12+4$ bytes are added to packets from the application layer; the resulting blocks are fragmented in payloads of MPEG2-TS packets that are managed at layer 2 according to the Multi Frequency-Time Division Multiple Access (MF-TDMA) scheme, allowing an RCST to transmit at a rate up to $2 \mathrm{Mbit} / \mathrm{s}$.

MF-TDMA is a multiple access technique where RCSTs transmit their data using a range of carrier frequencies (with potentially different bandwidth size), each of them organized in super-frames, frames and time-slots. Time-slots characteristics can be fixed (Figure 2(a)) or dynamic (Figure 2(b)). In the former case, time slots have fixed characteristics, in terms of bandwidth and duration. Whereas, in the latter case, besides bandwidth and time-slot duration, both transmission rate and code rate can be changed in consecutive slots. We will refer to a fixed slot structure.

At layer 1, MPEG2-TS packets are processed in several steps, such as: channel encoding (outer Reed-Solomon coding, convolutional interleaver, inner convolutional encoding, puncturing), base-band shaping of impulses, and Quadrature Phase Shift Keying (QPSK) modulation.

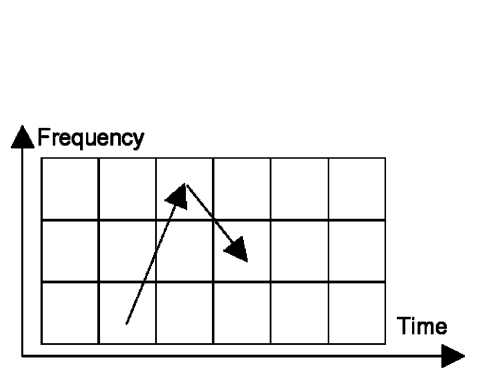

(a)

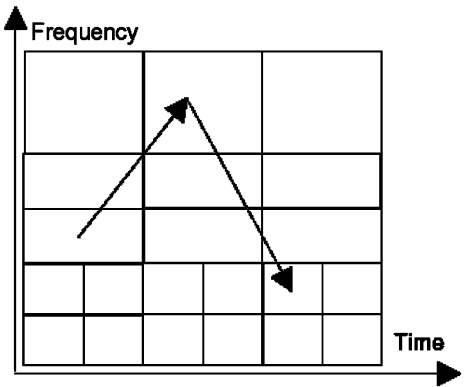

(b)

Figure 2. (a) MF-TDMA with fixed slot structure. (b) MF-TDMA with dynamic slot structure. 
An RCST can have assigned slots belonging to the same frequency or to different frequencies with the constraint that only one frequency can be transmitted by an RCST at the same time instant (Figure 2). According to the DVB-RCS standard, RCSTs send their resource requests to the NCC. The NCC, after having checked availability of return link resources, assigns to each RCST a group of bursts (i.e. the related slots), each of them characterized by frequency, bandwidth, start time and duration. In particular, it communicates resource allocations to RCSTs on the forward channel through the Terminal Burst Time Plan (TBTP), a control message belonging to the set of Service Information (SI) tables.

The DVB-RCS standard allows fixed or dynamic time slot allocations according to the five different schemes described below:

- Continuous Rate Assignment (CRA): CRA is a fixed and static allocation of resources after an initial set-up phase with a negotiation between the RCST and the NCC. With CRA, a given number of time slots are continuously assigned to an RCST every super-frame until that RCST sends the assignment release message.

- Rate-Based Dynamic Capacity (RBDC): RBDC is rate capacity dynamically requested by the RCST to the NCC. Each request is absolute, corresponding to the full rate currently being requested, overrides all previous RBDC requests from the same RCST, and shall be subject to a maximum rate limit directly negotiated between the RCST and the NCC.

- Volume-Based Dynamic Capacity (VBDC): VBDC is volume capacity dynamically requested by the RCST to the NCC. These requests are cumulative (i.e. each request shall add to all previous ones from the same RCST), indicating a total number of traffic slots that are needed to transmit all data currently available in the MAC queue. These slots can be shared among several super-frames. MAC parameters are the minimum and the maximum VBDC capacity that can be assigned to an RCST. VBDC is the default mode in DVB-RCS.

- Absolute Volume-Based Dynamic Capacity (AVBDC): AVBDC is volume capacity dynamically requested by the RCST to the NCC; AVBDC requests are absolute (i.e. each request replaces the previous ones from the same RCST) and indicate a total number of traffic slots that can be assigned in several super-frames. AVBDC is used for loss recovery in the VBDC mode.

- Free Capacity Assignment (FCA): FCA is volume capacity that shall be assigned to RCSTs from capacity that would be otherwise unused by CRA, RBDC and (A)VBDC methods. The term 'free' in FCA refers to 'spare' system capacity. Such a resource assignment shall be automatic, not involving any request from the RCST. Capacity assigned in this category is intended as a bonus capacity that can be used to reduce delays for any traffic type that can tolerate delay jitter.

Every super-frame, slots are assigned by the NCC in an orderly manner on the basis of both the request category (i.e. CRA $>$ RBDC $>(A) V B D C>$ FCA) and the Service Level Agreements (SLAs) between operator and customers. Resource allocation to RCSTs is performed throughout the TBTP message.

In DVB-RCS, BoD is supported by standard Demand-Assignment Multiple Access (DAMA) schemes, such as RBDC, VBDC, and AVBDC. In these cases, the NCC assigns capacity as a response to explicit requests made by RCSTs. These methods permit an efficient management of the shared resources, but the signalling exchange needed to reserve capacity entails an additional 


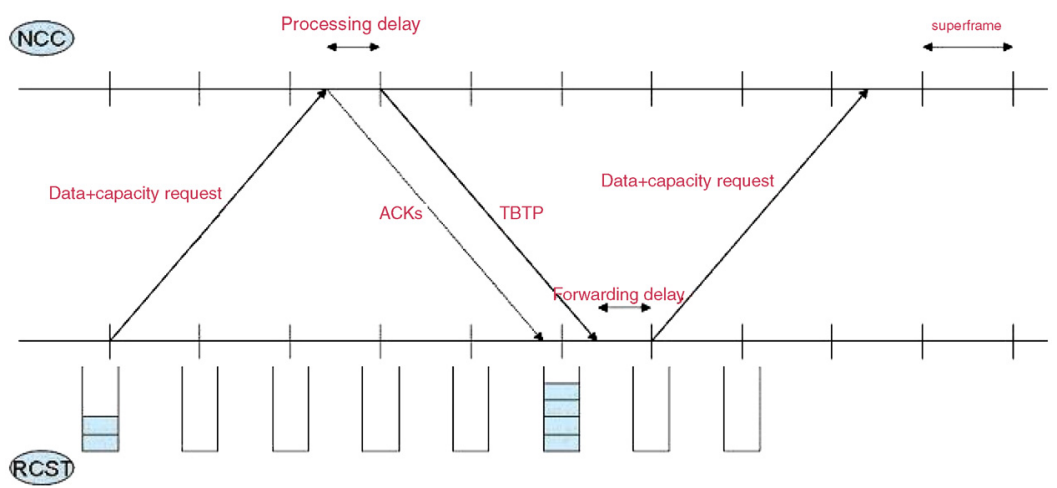

Figure 3. Description of access delay contributions (DAMA allocations).

end-to-end delay contribution, called access delay, that affects the transport layer performance. The access delay is composed of (Figure 3):

- The reservation delay: since requests are sent at a fixed rate in dedicated slots, a time interval occurs between the arrival of data in the MAC buffer and the transmission of the corresponding capacity request;

- A round trip propagation delay (sum of the time to propagate the capacity request from the RCST to the NCC and the time to deliver the TBTP in the opposite direction);

- The processing (and synchronization) delay: time spent by the DAMA controller (in the NCC) to transmit the TBTP message with the capacity assignment;

- The forwarding delay: time between the reception of a TBTP and the effective transmission of data.

Due to the access delay of standard DAMA schemes, we have proposed in this paper an innovative BoD approach detailed in Section 5.

\section{TCP MECHANISMS}

TCP is a transport protocol that provides a reliable, byte stream-based and connection-oriented service to the application layer. Three functions can be identified in the TCP protocol: flow control, congestion control and error recovery. The flow control scheme allows an efficient exchange of data not exceeding the capacity of the receiver buffer; it is based on the advertised window, rwnd, indicating the free space on the receiver buffer [12]. The congestion control scheme governs how TCP avoids and reacts to network congestion. In particular, TCP uses a variable, called congestion window (cwnd), to control the amount of data sent at a certain time. Therefore, the network status is probed by gradually increasing cwnd until congestion is experienced and cwnd is resized [1]. The minimum between cwnd and rwnd determines the transmission window. TCP uses cumulative acknowledgements (ACKs) to notify the sender on the last segment correctly received and in order [12]. Then, the timeout expiration at the sender or the receipt of duplicated ACKs indicates the loss of the corresponding segment, thus triggering one of the implemented error recovery schemes. TCP cannot discriminate between 
congestion losses and corruption losses. Hence, TCP considers the segment loss due to errors in the radio channel as an indication of network congestion, thus reducing its cwnd value. Such an assumption causes dramatic effects on the TCP performance (i.e. a significant and sudden goodput reduction) over wireless links.

In the following analysis, we have considered the standard TCP NewReno version that is largely diffused in the current operating systems and it is well suited to manage efficiently multiple packet losses in a window of data, a typical situation in wireless systems [13]. Its congestion control scheme is based on four main algorithms, named: Slow Start (SS), Congestion Avoidance (CA), Fast Retransmit and Fast Recovery [1]. The SS phase runs either at the start of the connection or after timeout expirations, performing an exponential increase in cwnd every time the TCP sender receives an ACK for the sent data. TCP triggers the CA mechanism when cwnd gets through a slow start threshold (ssthresh) value, or after the Fast Retransmit/Fast Recovery phase, allowing a gentler probing of the available capacity by a linear increase of cwnd. Note that the TCP response time is on a Round Trip Time (RTT) basis, thus entailing a very slow process of traffic injection increase in a satellite network scenario.

\section{CROSS-LAYER AIR INTERFACE DESIGN}

\subsection{Motivations and available approaches}

The current Internet design is based on a layered protocol stack (similar to that envisaged by the Open System Interconnection (OSI) reference architecture) that permits to solve independently distinct problems at different levels. Such a separation leads to a natural abstraction, enabling heterogeneous networks to be efficiently interconnected.

The changes in the communication features due to time-varying radio channel behaviours for mobile users, diverse user devices, autonomous networks and software dependence can entail a sub-optimal efficiency of the layered protocol approach. In general, wireless networks (e.g. $\mathrm{WiFi}$, ad hoc, satellite, etc.) introduce further 'factors' to be considered for achieving performance optimization, such as power availability, radio channel conditions, etc. In order to address these problems, an innovative cross-layer approach could be adopted in the protocol architecture design, guaranteeing to share information among non-adjacent layers for largescale system adaptivity.

For instance, the basic TCP protocol assumption that lost packets are necessarily due to network congestion may be not applicable in wireless systems where time-varying channel conditions can cause the corruption of TCP packets. In this case, a cross-layer signalling exchange, where lower layers notify the transport layer about the radio channel conditions, may allow TCP to take more appropriate decisions in setting the cwnd value and, hence, the transmission rate.

The communication between non-adjacent layers can be performed with different methods. Below, three different approaches to support 'vertical' interactions among protocols are mentioned according to [14]:

- Packet headers: In IPv6, additional headers can be used to carry information by in-band message carriers (Interlayer Signalling Pipe, ISP).

- ICMP Messages: Dedicated internal messages can be exchanged by creating appropriate holes in the protocol stack. 
- Management by 'third parties': Internal or external 'entities' can store protocol state information and use such knowledge to modify the state of non-adjacent layers.

\subsection{Dynamic resource allocation for TCP flows}

Let us refer to a BoD allocation scheme to support TCP-based flows. In this case, both MAC protocol (resource request-resource allocation loop of the BoD technique) and TCP (cwndACK loop) evolve on a round-trip propagation delay basis. In order to overcome the loss of efficiency due to these combined delays, we propose here a BoD scheme that uses cross-layer interactions between layer 4 and layer 2 to optimize the resource management and to reduce the access delays due to the nested control loops in TCP and MAC layers (Figure 4). While in the classical VBDC scheme, an RCST dynamically requests the total volume of slots needed to empty its MAC queue, we consider a TCP-driven BoD scheme (of the VBDC type), where an RCST makes a preventive request for the data that the TCP level will provide at the MAC queue in the next RTT.

In particular, we consider a cross-layer signalling method based on the same guidelines used in the definition of the Cross-Layer Signalling Shortcuts (CLASS) scheme in Reference [14]. That is a simple approach, where TCP internal status information in the RCST is propagated from layer 4 to layer 2 through local out-of-band shortcuts, using dedicated Application Programming Interfaces (APIs); a light-weighted internal signalling format is used to keep the overhead to just a minimum. Once arrived at layer 2, cross-layer information regarding both the estimated value of cwnd $_{\text {next }}$ in the next RTT and the TCP state (SS or CA) is encapsulated into the MAC header** ${ }^{\dagger \dagger}$ of the packet sent by the RCST.

The NCC examines the header of any incoming packet in order to compare the cwnd $\mathrm{d}_{\text {next }}$ value with the amount of resources already assigned $\left(a_{\text {res }}\right)$ to the corresponding RCST. Two cases are possible:

1. If $\mathrm{cwnd}_{\text {next }}<a_{\text {res }}$, the NCC considers that a packet loss has occurred so that the cwnd has

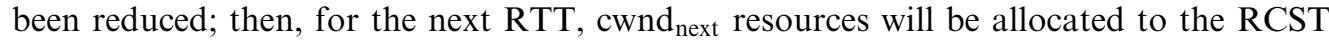
and $a_{\text {res }}-$ cwnd $_{\text {next }}$ resources will be made available to satisfy the needs of the connections of other RCSTs.

2. If cwnd $\mathrm{next}_{\mathrm{n}}>a_{\mathrm{res}}$, the NCC considers that the RCST needs a capacity increase in the next RTT. At the NCC, the corresponding request is then inserted either in a high-priority queue, if occurring in the SS phase, or in a low-priority one, if occurring in the CA phase, as specified by the TCP phase flag in the MAC header. The requests in the queues are served as follows:

2.1. The NCC allocates further resources (in the next RTT through the broadcast TBTP message) first to requests in the high-priority queue and then to requests in the lowpriority queue (if there is still available capacity).

\footnotetext{
"* In the presence of encryption, we have a particular case for our TCP-driven resource allocation scheme, since the sender address is hidden. A possible solution could be to copy such information into the MAC header that also contains the estimated cwnd value to be sent to the NCC. Further details on this topic are beyond the scope of this paper.

$\dagger$ Note that the classical 'capacity request' messages foreseen in the DVB-RCS standard are not viable, since they contain only a limited information. We need to use enriched and optimized headers in the layer 2 packets to convey requests to the NCC.
} 
2.2. A minimum resource allocation of one slot per super-frame is granted to RCSTs starting to transmit (SS phase). When all resources are already assigned, a new RCST entering the service will receive a minimum resource allocation by taking it from the RCST with the highest amount of assigned resources.

2.3. When new resources are available (due to the end of a connection or due to a cwnd reduction) these are primarily assigned to RCSTs in the SS phase (high-priority queue).

2.4. Within each queue the available resources are assigned to all the pending requests according to the Maximum Legal Increment (MLI) algorithm [15] that guarantees a fair approach.

2.5. When all the resources are assigned (the system is congested), the BoD scheme envisages a fair mechanism that de-allocates resources on a super-frame basis from those TCP flows with higher assigned resources in favor of those with lower resources (irrespective of the related TCP flow phases) in order to avoid that older TCP flows saturate the available capacity and do not allow newcomers to acquire adequate resources.

If the amount of needed resources exceeds those available in a super-frame, the NCC creates a waiting list to assign the resources in the next super-frames and stops the cwnd growth of all the connections coming from the RCSTs that have not (or just partly) obtained the requested resources. In particular, the NCC performs a further cross-layer action that sets a new variable (lower than the current cwnd $_{\text {next }}$ value), named cwnd*, in order to modify the current cwnd value used by the TCP source as: cwnd $\leftarrow$ cwnd*. Note that the NCC sends back the cwnd* value by using a field for TCP options (layer 4 ACKs) in the headers. Such cwnd lock procedure is operated for all the time that resources are saturated; hence, during congestion periods, a TCP source has a flat cwnd behaviour forced by layer 2 of the NCC. The rationale of this modification on the TCP protocol is to avoid internal congestion at the RCST side and, then, the possibility of buffer overflows. This approach is quite important: since the $\mathrm{NCC}$ has a complete control over network resources, it can stop (in the presence of congestion) further increases of cwnd that would only cause packet losses and throughput reduction at the TCP level.

\subsection{Algorithm description}

Let $\Gamma$ denote the set of admitted RCSTs, $\Gamma=\{1,2, \ldots, m\}$, being $m$ the number of RCSTs. Let $\Theta$ represent the set of RCSTs in SS phase and $\Omega$ the set of RCSTs in CA phase. Note that $\Gamma=\Theta \cup \Omega$. Let $s$ denote the number of slots per super-frame and $c$ denote the number of carriers. Since an RCST cannot have assigned two simultaneous slots on different frequencies, the actual number of assignable resources (i.e. slots) in a super-frame, $P$, is defined as

$$
P=\min [m, c] \times s
$$

Hence, on the basis of (1), the number of available resources in a super-frame, $A$, is

$$
A=P-\Sigma_{i \in \Gamma}\left\{a_{\mathrm{res}}(i)\right\}
$$


Table I. Pseudo-code description of the cross-layer BoD scheme.

Step 0: Call Admission Control (CAC)

If $\mathrm{Ris}_{\min } \leqslant A$ when the new RCST $l$ accesses the network

Then $a_{\mathrm{res}}(l)=\operatorname{Ris}_{\min }$ and $\Gamma=\Gamma+\{l\}$

Else $k=\arg \max _{h \in \Gamma}\left[a_{\text {res }}(h)\right]$

If $a_{\text {res }}(k)-\mathrm{Ris}_{\min } \geqslant \mathrm{Ris}_{\text {min }}$

Then

$a_{\mathrm{res}}(k)=a_{\mathrm{res}}(k)-\operatorname{Ris}_{\min }, a_{\mathrm{res}}(l)=\operatorname{Ris}_{\min }, \Gamma=\Gamma+\{l\}$

Else The new RCST is blocked

Step 1: RCST side (return channel, top-down scheme), TCP-based resource request phase

$\forall i \in \Gamma$ If $\operatorname{cwnd}(i)<\operatorname{ssthresh}(i)$

Then $\operatorname{cwnd}_{\text {next }}(i)=2 \times \operatorname{cwnd}(i)$

Else $\operatorname{cwnd}_{\text {next }}(i)=\operatorname{cwnd}(i)+1$

Step 2: NCC side (forward channel, top-down scheme), fair resource allocation

If $\Sigma_{i \in \Gamma}\left\{\mathrm{cwnd}_{\text {next }}(i)\right\} \leqslant A$ (here $A$ is related to the resource allocation made in the previous super-frame)

Then $\forall i \in \Gamma\left\{\right.$ new_res $(i)=\operatorname{cwnd}_{\text {next }}(i)-a_{\text {res }}(i)$

$a_{\text {res }}(i)=a_{\text {res }}(i)+$ new_res $\left.(i)\right\}$

$\forall i \in \Gamma \operatorname{cwnd}^{*}(i)=a_{\mathrm{res}}(i)$

If $A=0$ (here $A$ is related to the resource allocation made in the previous super-frame)

$$
\begin{array}{r}
\forall i \in \Gamma, \forall j \in \Gamma \text { If } \text { cwnd }_{\text {next }}(j) \neq \operatorname{cwnd}_{\text {next }}(i) \\
\text { Then }\left\{\forall n \in \Theta \text { new_res }(n)=\operatorname{cwnd}_{\text {next }}(n)-a_{\text {res }}(n)\right. \\
k=\arg \max _{h \in \Omega}\left[a_{\text {res }}(h)\right] \\
a_{\text {res }}(k)=a_{\text {res }}(k)-\text { new_res }(n) \\
\left.a_{\text {res }}(n)=a_{\text {res }}(n)+\text { new_res }(n)\right\}
\end{array}
$$

Before Step 3, NCC must wait for the processing delay.

Step 3: RCST side (forward channel, bottom-up scheme), TCP congestion window update

$\forall i \in \Gamma \operatorname{cwnd}(i)=\min \left[\operatorname{cwnd}_{\text {next }}(i), \operatorname{cwnd}^{*}(i)\right]$

After Step 3 (and before the iteration of the algorithm) RCST must wait for the forwarding delay.

We can note that a new RCST, that starts to transmit, needs to have assigned a minimum resource $\left(\mathrm{Ris}_{\min }\right)$ of one slot per super-frame. Note that $\mathrm{Ris}_{\min }$ is not explicitly requested by the RCST, but it is allocated by the NCC in the set-up connection phase to permit to each RCST to send initially some packets. In general, $\mathrm{Ris}_{\min }$ is a part of the above-mentioned $a_{\text {res }}$ value

$$
a_{\text {res }}=\text { Ris }_{\min }+\text { Ris }_{\text {ext }}
$$

where Ris ext represents the extra resources (with respect to the minimum $\mathrm{Ris}_{\min }$ ones) that are assigned to the RCST.

The detailed pseudo-code description of our TCP-based BoD algorithm is provided in Table I by using different state variables. 


\section{SIMULATION RESULTS}

A simulation platform, modelling a DAMA scheme, has been implemented using the satellite extensions of the Network Simulator ns-2 (v. 2.27) [16]. In particular, our examined scenario is characterized as follows:

- A GEO bent-pipe satellite connecting multiple RCSTs with the NOC;

- A grid of 'nodes' on the Earth, defined by their latitude and longitude;

- A satellite interface stack to connect nodes to satellite channel;

- One (or more) TCP agents attached to each node generating data traffic, so that the RCST behaviour is obtained;

- Uncorrelated packet losses have been assumed on the satellite links (additive white Gaussian noise links represent a reasonable assumption in case of GEO satellites with fixed terminals).

Moreover, our simulator contains a $\mathrm{C}++$ code in order to obtain: a centralized MF-TDMA allocation scheme, cross-layer interactions and the NCC functionalities (including the proposed BoD scheme). In practice, we have extended the internal ns-2 code, replacing the 'old' MAC model, based on Aloha, with a 'new' module able to schedule the transmission time of packets on the basis of an MF-TDMA tunable frame structure. Moreover, since synchronization, acquisition, and logon signalling (all these procedures are managed on a frame basis) are out of the scope of this paper, we consider, a correspondence between frame and super-frame $[3,4]$. Then, we have created particular 'tcl' procedures to perform cross-layer tasks and MAC scheduling. Our simulation also entails transport and application layers. The main simulation parameters are detailed in Table II.

In the depicted scenario, we have performed a wide simulation campaign aimed at showing how TCP dynamics benefit by the cross-layer interaction with the MAC layer. To this purpose, we have considered several RCSTs, each of them with an FTP transfer, that share the limited return link capacity. Our performance evaluation has been organized in three steps:

1. Analysis of the dynamic assignment/removal of resources in the return link when the cross-layer-based allocation scheme is employed (Section 6.1);

2. Performance comparison with a fixed allocation scheme (Section 6.2);

3. Performance comparisons with the classical BoD schemes defined by the DVB-RCS standard (Section 6.3).

Table II. Main simulation parameters.

End-to-end round trip propagation delay

Return link bandwidth (shared among RCSTs)

Super-frame duration

Packet error rate, PER

TCP packet size

Transport protocol

Application protocol

Maximum number of TCP sources (i.e. RCSTs)

File size

$\sim 512 \mathrm{~ms}$
$2 \mathrm{Mbit} / \mathrm{s}$
$96 \mathrm{~ms}$
$\left[\right.$ Error free; $10^{-4}, 10^{-3} ; 5 \times 10^{-3}$ ]
1500 bytes
TCP NewReno
FTP
32
10 Mbytes




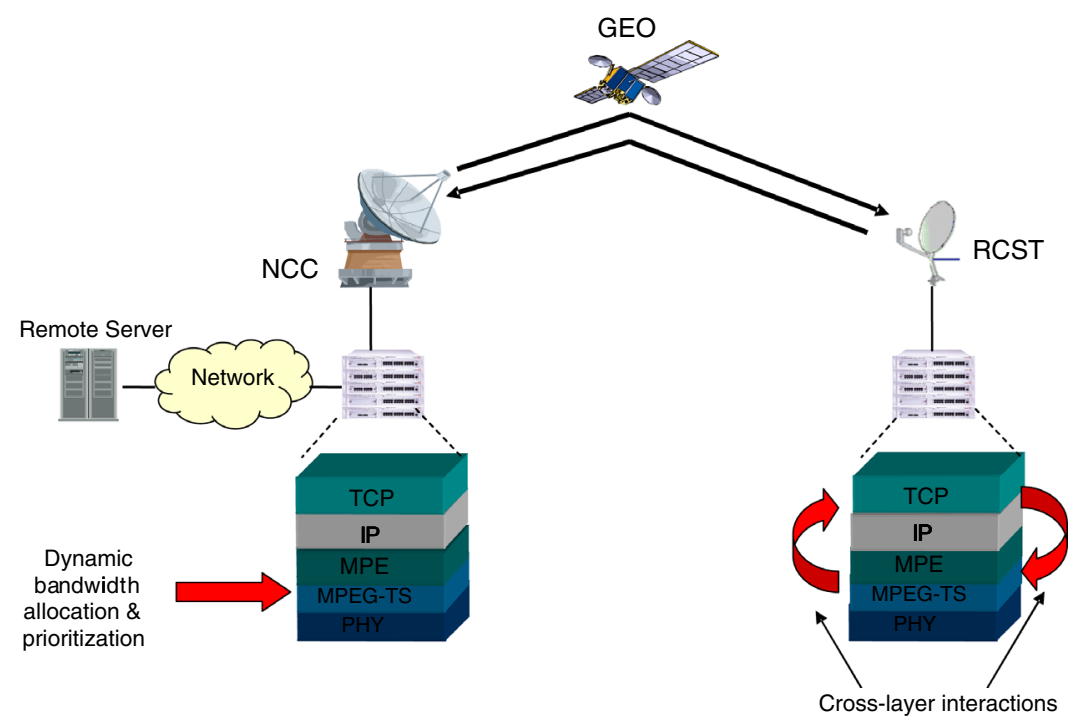

Figure 4. Protocol stack and cross-layer interactions (view of both the RCST side and the NCC side).

\subsection{Analysis of the proposed cross-layer resource allocation scheme}

In Figure 5, we have monitored the assigned resources as a function of time with respect to the current TCP cwnd value in the presence of Packet Error Rate, PER $=5 \times 10^{-4}$; in this case, there is just one TCP connection in the system. These results highlight how the allocated resources (measured in terms of equivalent ${ }^{\text {th }} \mathrm{TCP}$ segments) are strictly related to cwnd variations: exponential increase during the SS phase, linear increase during the CA phase and halving when the Fast Retransmit/Fast Recovery mechanisms are invoked as reaction to a segment loss. Note that the cwnd behaviour in Figure 5 is affected by packet losses with consequent cwnd halving; moreover, due to the presence of random errors, congestion does not occur.

Figure 6 shows the resources allocated to two TCP connections starting at different time instants and affected by transmission errors $\left(\mathrm{PER}=1.5 \times 10^{-4}\right)$. The main characteristics of the allocation process are as follows:

- At the beginning, NCC serves connection \#1 in an exclusive mode: resources (measured in equivalent TCP segments) are assigned according to the TCP cwnd growth;

- A fair sharing of resources is performed when connection \#2 starts. In fact, after the start up phase the capacity allocation is perfectly shared between the two TCP flows;

- When saturation occurs, NCC stops the cwnd increase of both connections in order to prevent congestion and, then, higher delays;

- When one connection is affected by errors (with consequent cwnd reduction), NCC reassigns temporarily the unused capacity to the other connection.

\footnotetext{
Note that, according to the simulation parameters listed in the Table II, the super-frame is able to transport the equivalent of about 110 TCP segments.
} 


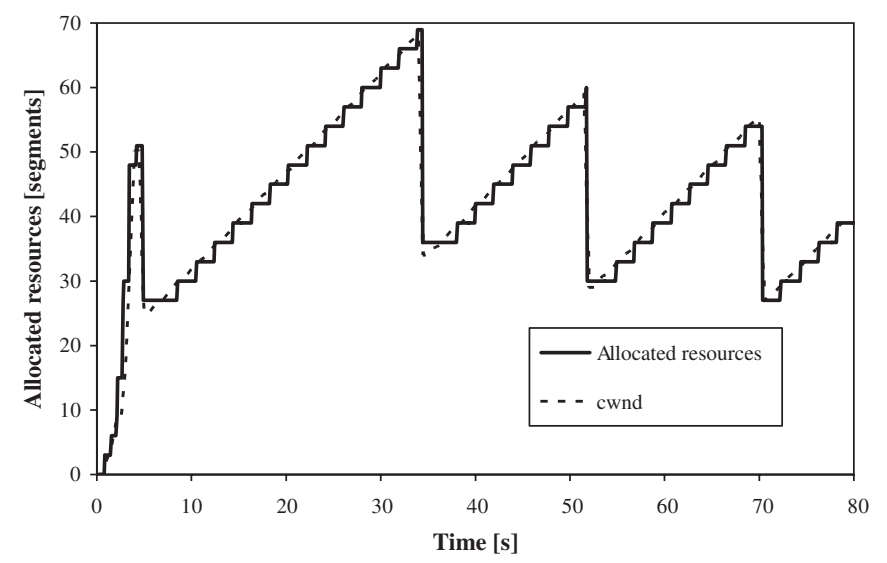

Figure 5. Comparison among allocated resources and cwnd trend vs time ( 1 TCP connection, $\left.\mathrm{PER}=5 \times 10^{-4}\right)$.

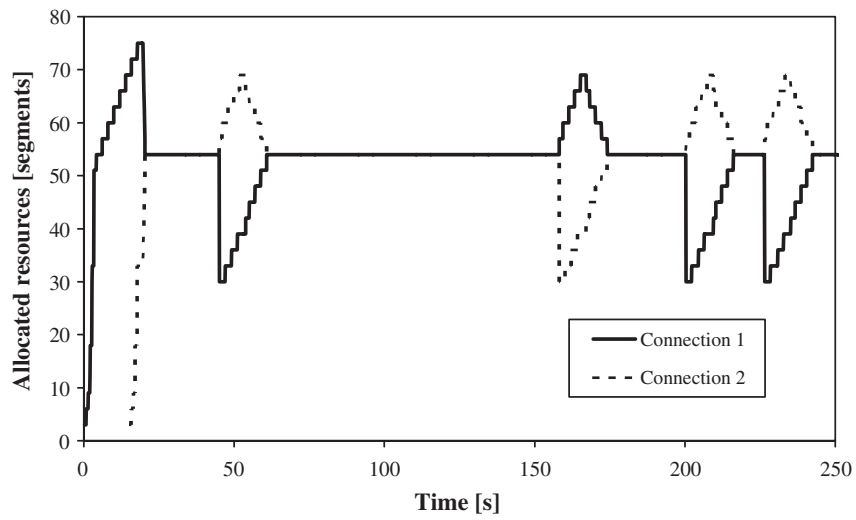

Figure 6. Comparison among allocated resources vs time (2 TCP connections, PER $=1.5 \times 10^{-4}$ ).

\subsection{TCP-driven BoD versus fixed allocation scheme}

Referring to a scenario with multiple TCP connections, this Section compares the proposed TCP-driven BoD scheme with a fixed allocation scheme (i.e. CRA) where the available return link capacity of $2 \mathrm{Mbit} / \mathrm{s}$ is equally shared by the connections; our aim is to evaluate the improvement in the end-to-end TCP performance.

Let us refer to a case with 20 RCSTs accessing the return link to download 10 Mbytes files from a remote server by means of the FTP application protocol. These file transfers start at time instants spaced of $5 \mathrm{~s}$. The average file transfer time has been shown for different PER values in Figure 7. These results show that the proposed cross-layer BoD scheme achieves a better performance and that such improvement increases with PER. For instance, the average file transfer time is reduced of $12.3 \%$ in the error-free case (up to about $26.5 \%$ for PER $=10^{-2}$ ) with our BoD scheme with respect to the fixed allocation case. In conclusion, our cross-layer method allows a better reaction to dynamic changes in the transmission rate by distributing (and 


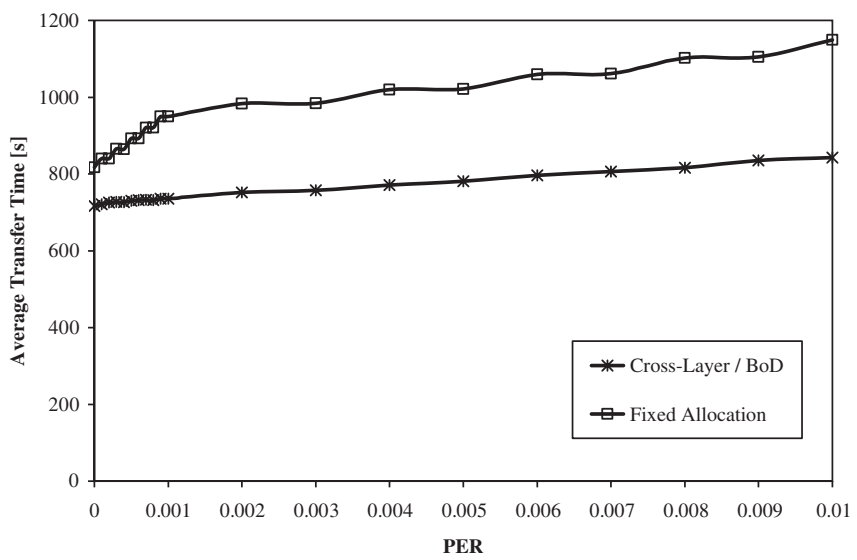

Figure 7. Average transfer time vs PER (20 TCP connections starting at instants spaced of $5 \mathrm{~s}$ ).

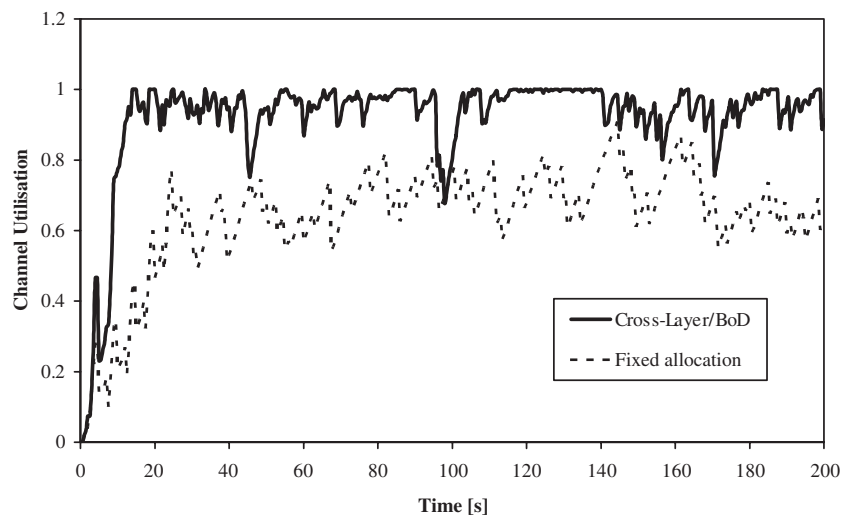

Figure 8. Channel utilization vs time (5 TCP connections starting at instants spaced of $5 \mathrm{~s}, \mathrm{PER}=10^{-3}$ ).

re-distributing) the capacity on the basis of the current needs of competing TCP flows. This result is confirmed by Figure 8 that traces the channel utilization over the time in the case that 5 FTP transfers are performed starting at time instants spaced of $5 \mathrm{~s}$.

\subsection{TCP-driven BoD versus standard BoD schemes}

In the case of a single connection, this Section compares our TCP-driven BoD technique with VBDC assignment and an implementation of the RBDC allocation scheme. As for the RBDC algorithm, we consider that the BoD agent in the RCST computes its resource request every super-frame by equalling input and output data rate for the MAC buffer [17]. The absolute rate request of $\mathrm{RBDC}$ has been expressed as follows in terms of equivalent capacity of bytes per super-frame:

$$
R_{\mathrm{RBDC}}(k)=R_{\mathrm{RBDC}}(k-1)+[Q(k)-Q(k-1)]
$$


where $R_{\mathrm{RBDC}}(k)$ and $R_{\mathrm{RBDC}}(k-1)$ are, respectively, the requests at the $k$ th and $(k-1)$ th superframes; moreover, $Q(k)$ and $Q(k-1)$ represent the MAC queue size at the beginning of the $k$ th and $(k-1)$ th super-frames, respectively.

Figure 9 shows the average perceived RTT values for the three BoD schemes considered: VBDC leads to the highest mean RTT values, while RBDC and the cross-layer-based scheme achieve better performance. These results can be justified as follows. RBDC maintains a fixed capacity allocation for the super-frame duration; such capacity is frame-by-frame updated on the basis of requests; this means that in the steady-state a certain capacity is available for an RCST and that only variations experience the access delay. Whereas, VBDC individually requests the allocation of capacity for the new packets arrived in the super-frame so that each transmission request for a group of packets is served with an access delay. This is the reason why higher delays are perceived by traffic with VBDC than with RBDC. Finally, our cross-layer BoD scheme outperforms the RBDC technique since it has an exact knowledge of the future traffic behaviour due to the TCP protocol (it can anticipate TCP needs). In conclusion, referring to Figure 9, our BoD scheme achieves a $60 \%$ reduction of the mean RTT value with respect to VBDC and a $15 \%$ reduction with respect to RBDC.

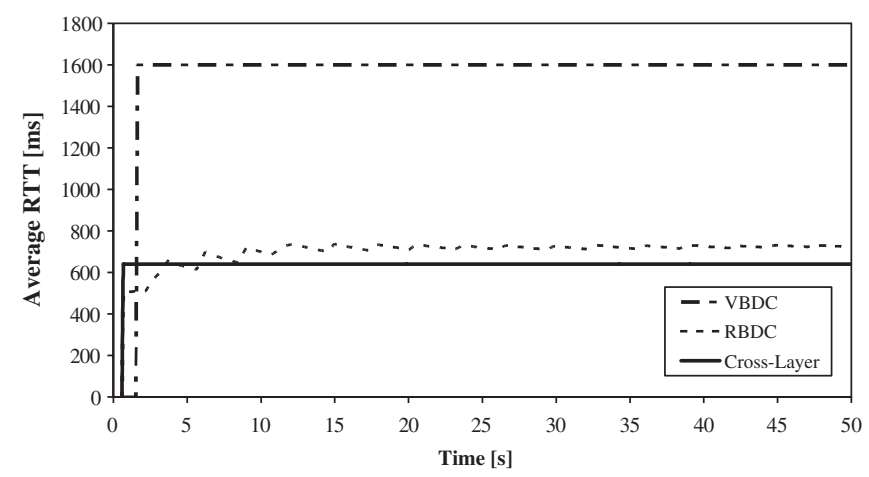

Figure 9. Comparison among average RTT values vs time (VBDC, RBDC and cross-layer access schemes).

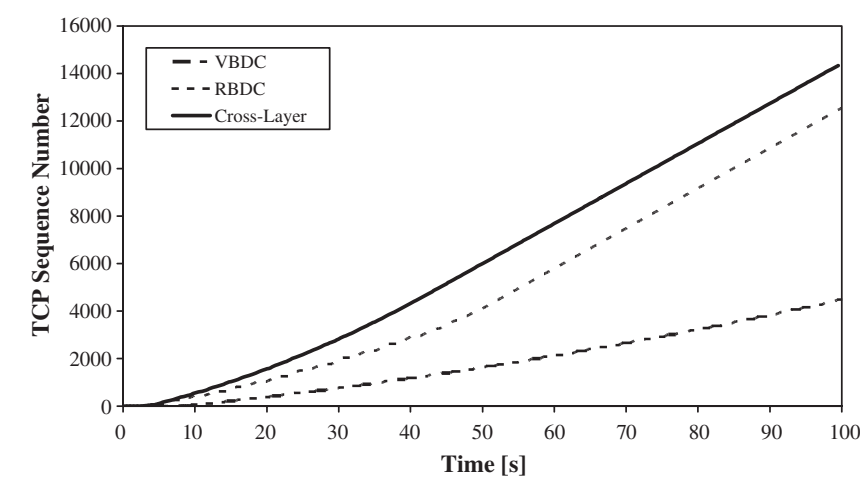

Figure 10. TCP sequence number vs time (VBDC, RBDC and cross-layer access schemes). 
Since the TCP response time depends on the RTT, a relationship of inverse proportionality occurs between the perceived delay at layer 4 and the end-to-end performance. To this purpose, Figure 10 traces the sequence number of the received packets as a function of time. After $100 \mathrm{~s}$ of transmission, the number of packets transmitted by the proposed $\mathrm{BoD}$ scheme is 3 times greater than that obtained by using VBDC and about $15 \%$ greater than that obtained by considering the RBDC technique.

\section{CONCLUSIONS}

The resource allocation scheme used in the return link of a DVB-RCS network plays a fundamental role for the achievement of a good performance when TCP flows compete for the shared resources. A fixed allocation scheme (i.e. CRA) leads to non-optimal network utilization since TCP implements flow control and congestion control that dynamically regulate the transmission rate on an RTT basis. In fact, as long as the TCP window is smaller than the bandwidth-delay product, part of the assigned capacity will be unused. On the other hand, standard DAMA schemes guarantee an optimal network utilization, but impacting TCP endto-end performance and also introducing additional delay contributions to the RTT due to the signalling exchange (request/allocation process).

The proposed allocation scheme aims both to allocate dynamically resources and to limit the access delay through a cross-layer interaction between TCP and MAC layers. On the basis of the TCP internal state, a prediction is made for the amount of data that will feed the MAC buffer in the next RTT in order to request in advance the needed transmission resources.

Simulation results show that the proposed BoD scheme permits to follow accurately the cwnd evolution and allows a fair resource sharing among competing flows. Moreover, since the available resources are better utilized, there is a reduction in the mean file transfer delay with the FTP application (elephant connections), compared with a fixed allocation scheme. Finally, we have proved that our cross-layer-based $\mathrm{BoD}$ scheme presents a lower average perceived RTT and a higher number of transmitted packets per time unit with respect to VBDC and RBDC.

\section{NOMENCLATURE}

$\begin{array}{ll}\text { ACK } & \text { Acknowledgement } \\ \text { API } & \text { Application Programming Interface } \\ \text { AVBDC } & \text { Absolute Volume-Based Dynamic Capacity } \\ \text { BDP } & \text { Bandwidth Delay Product } \\ \text { BoD } & \text { Bandwidth on Demand } \\ \text { BSS } & \text { Broadcast Satellite Service } \\ \text { CA } & \text { Congestion Avoidance } \\ \text { CAC } & \text { Call Admission Control } \\ \text { CRA } & \text { Continuous Rate Assignment } \\ \text { CRC } & \text { Cyclic Redundancy Check } \\ \text { cwnd } & \text { congestion window } \\ \text { DAMA } & \text { Demand Assignment Multiple Access } \\ \text { DTH } & \text { Direct-To-Home }\end{array}$




$\begin{array}{ll}\text { DVB-RCS } & \text { Digital Video Broadcasting-Return Channel via Satellite } \\ \text { DVB-S } & \text { Digital Video Broadcasting-Satellite } \\ \text { FCA } & \text { Free Capacity Assignment } \\ \text { FSS } & \text { Fixed Satellite Service } \\ \text { FTP } & \text { File Transfer Protocol } \\ \text { GEO } & \text { Geosynchronous (Geostationary) Earth Orbit } \\ \text { ICMP } & \text { Internet Control Message Protocol } \\ \text { IP } & \text { Internet Protocol } \\ \text { ISN } & \text { Interactive Satellite Network } \\ \text { MAC } & \text { Medium Access Control } \\ \text { MF-TDMA } & \text { Multi Frequency-Time Division Multiple Access } \\ \text { MLI } & \text { Maximum Legal Increment } \\ \text { MPE } & \text { Multi-Protocol Encapsulation } \\ \text { NCC } & \text { Network Control Center } \\ \text { NOC } & \text { Network Operation Center } \\ \text { OSI } & \text { Open System Interconnection } \\ \text { PER } & \text { Packet Error Rate } \\ \text { QoS } & \text { Quality of Service } \\ \text { QPSK } & \text { Quadrature Phase Shift Keying } \\ \text { RBDC } & \text { Rate-Based Dynamic Capacity } \\ \text { RCST } & \text { Return Channel Satellite Terminal } \\ \text { RRM } & \text { Radio Resource Management } \\ \text { RTT } & \text { Round Trip Time } \\ \text { rwnd } & \text { receiver advertised window } \\ \text { SI } & \text { Service Information } \\ \text { SLA } & \text { Service Level Agreement } \\ \text { SS } & \text { Slow Start } \\ \text { ssthresh } & \text { slow start threshold } \\ \text { TBTP } & \text { Terminal Burst Time Plan } \\ \text { TCP } & \text { Transmission Control Protocol } \\ \text { TS } & \text { Transport Stream } \\ \text { VBDC } & \text { Volume-Based Dynamic Capacity } \\ \text { WiFi } & \text { Wireless Fidelity } \\ & \end{array}$

\section{ACKNOWLEDGEMENTS}

This work was funded by the European Commission in the framework of the ja2430 activity of the SatNEx Network of Excellence project (EU IST FP6) - Contract No. 507052. Part of this work has been presented at the IWSSC 2005 Conference (5-9 September 2005), Siena, Italy.

\section{REFERENCES}

1. Stevens W. TCP Slow start, congestion avoidance, fast retransmit and fast recovery algorithms. IETF RFC 2001, January 1997.

2. Allman M, Glover D, Sanchez L. Enhancing TCP over satellite channels using standard mechanisms. IETF RFC 2488, January 1999. 
3. ETSI. Digital Video Broadcasting (DVB); Interaction Channel for Satellite Distribution Systems. EN 301790 , V1.3.1, 2003.

4. ETSI. Digital Video Broadcasting (DVB); Interaction Channel for Satellite Distribution Systems; Guidelines for the use of EN 301 790. TR 101790 , V1.2.1, 2003.

5. Kota SL. Broadband satellite networks: trends and challenges. IEEE Communications Society WCNC 2005; 3:14721478.

6. Guainella E, Pietrabissa A. TCP-friendly bandwidth-on-demand scheme for satellite networks. Proceedings of the ASMS Conference, Frascati, Rome, Italy, 10-11 July 2003.

7. Sooriyabandara M, Fairhurst G. Dynamics of TCP over BoD satellite networks. International Journal of Satellite Communication and Networking 2003; 21:427-449.

8. Yang S, de Veciana G. Size-based adaptive bandwidth allocation: optimizing the average QoS for elastic flows. Proceedings of IEEE INFOCOM 2002. The 21st Annual Joint Conference of the IEEE Computer and Communications Societies, New York, U.S.A., 23-27 June 2002.

9. Astuti D. TCP and link layer enhancements in DVB-S/DVB-RCS satellite systems. Report on line (date of access: April 28, 2006): www.cs.helsinki.fi/u/astuti/papers/TCP_and_Link_Layer_Enhancements_in_DVB-S_DVB-RCS_ satellite_systems.pdf.

10. Shakkottai S, Rappaport TS, Karlsson PC. Cross-layer design for wireless networks. IEEE Communications Magazine 2003; 41(10):74-80.

11. ETSI. Digital Video Broadcasting (DVB). Framing structure, channel coding and modulation for $11 / 12 \mathrm{GHz}$ satellite services. EN 300 421, V1.1.2, 1997.

12. Stevens W. TCP/IP Illustrated, vol. 1. Addison-Wesley: Reading, MA, 1994.

13. Floyd S, Henderson T. The NewReno modification to the TCP's fast recovery algorithm. IETF RFC 2582, April 1999.

14. Wang Q, Abu-Rgheff MA. Cross-layer signalling for next-generation wireless systems. IEEE Wireless Communications and Networking Conference, New Orleans, U.S.A., 16-20 March 2003.

15. Acar G, Rosenberg C. Algorithms to compute for bandwidth on demand requests in a satellite access unit. Proceedings of the 5th Ka-band Utilization Conference, Taormina, Sicily Island, Italy, October 1999; 353-360.

16. NS-2 Network Simulator (Vers. 2.27). URL (date of access: April 28, 2006): http://www.isi.edu/nsnam/ns/ nsbuild.html

17. Delli Priscoli F, Pietrabissa A. Control-theoretic bandwidth-on-demand protocol for satellite networks. Proceedings of IEEE Conference on Control Applications, Glasgow, U.K., September 2002.

\section{AUTHORS’ BIOGRAPHIES}

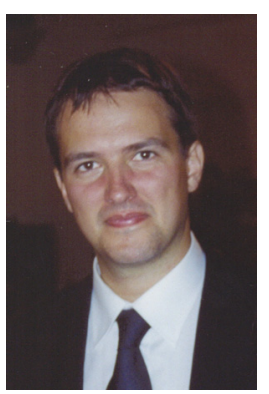

Paolo Chini was born in Siena, Italy, in 1972. He received the Dr Ing Degree in Telecommunications Engineering in 2005 from the University of Siena, Italy, with a thesis entitled 'Cross-layer management of resources in an interactive DVBRCS-based satellite network' in the framework of the SatNEx 'Satellite Communications Network of Excellence', in FP6 network of excellence, in cooperation with the University of Rome 'Tor Vergata'. From 2005 he performs research activities at the University of Siena; his fields of interests include satellite communications, DVBRCS standards, TCP and MAC layer protocols, mobile and wireless networks, HAPs platforms.

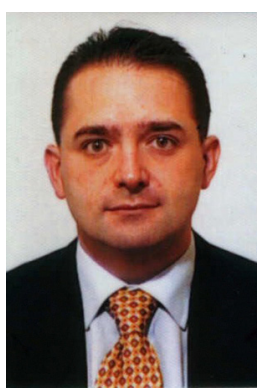

Giovanni Giambene was born in Florence, Italy, in 1966. He received the Dr Ing degree in Electronics from the University of Florence, Italy, in 1993 and the PhD degree in Telecommunications and Informatics from the University of Florence, Italy, in 1997. From 1994 to 1997, he was with the Electronic Engineering Department of the University of Florence, Italy. He was Technical External Secretary of the European Community COST 227 Action. He also contributed to the Resource Management activity of the Working Group 3000 within the RACE SAINT Project. From 1997 to 1998, he was with OTE of the Marconi Group, Florence, Italy, where he was involved in a GSM development program. In the same period he also contributed to the COST 252 Action research activities by studying the performance of Packet Reservation Multiple Access (PRMA) protocols suitable 
for supporting voice and data transmissions in low earth orbit mobile satellite systems. In 1999 he joined the Information Engineering Department of the University of Siena, Italy, first as research associate and then as assistant professor. He teaches the advanced course of Telecommunication Networks at the University of Siena. From 2000 to 2003, he contributed to the activities of the 'Personalised Access to Local Information and services for tOurists' (PALIO) IST Project within the fifth Research Framework of the European Commission (www.palio.dii.unisi.it). At present, he is involved in the SatNEx network of excellence (www.satnex.org) of the FP6 programme in the satellite field, as work package leader on radio resource management techniques (ja2330) and cross-layer air interface design (ja2230). He is also viceChair of the COST 290 Action (www.cost290.org). He has recently published a book entitled 'Queuing Theory and Telecommunications: Networks and Applications', Springer (May 2005). His research interests include third-generation mobile communication systems, medium access control protocols, traffic scheduling algorithms, and queuing theory.

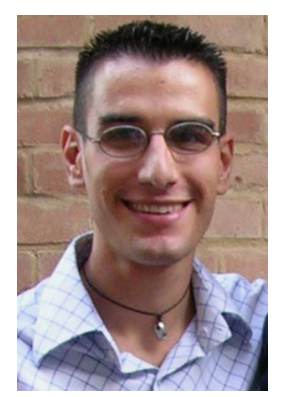

Danilo Bartolini graduated in Telecommunications Engineering in 2003 with a thesis entitled 'Algorithms of bandwidth on demand (BoD) for satellite networks' and obtained the Laurea degree in Telecommunications Engineering at University of Rome 'Tor Vergata' in 2005 with a thesis entitled 'TCP-MAC cross-layer interaction in the DVB-RCS satellite scenario'. From July to December 2005 he had a research grant within SatNEx (Satellite Communications Network of Excellence) to collaborate with the University of Rome 'Tor Vergata' on the study of satellite networks. His research interests cover broadband satellite networks, TCP/IP and MAC protocols.

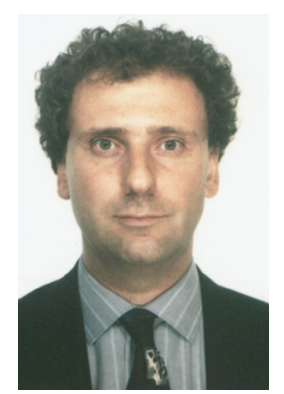

Michele Luglio received the Laurea degree in Electronic Engineering at University of Rome 'Tor Vergata'. He received the PhD degree in telecommunications in 1994. From August to December 1992 he worked, as visiting Staff Engineering at Microwave Technology and Systems Division of Comsat Laboratories (Clarksburg, MA, U.S.A.). He received the Young Scientist Award from ISSSE '95. From 1995 to 2004 he was research and teaching assistant at University of Rome 'Tor Vergata'. At present he is associate professor of telecommunication at the same university. $\mathrm{He}$ works on designing satellite systems for multimedia services both mobile and fixed, in the frame of projects funded by EC, ESA and ASI. He taught Signal Theory and collaborated in teaching Digital Signal Processing and Elements of Telecommunications. In 2001 and 2002 he was visiting Professor at the Computer Science department of University of California Los Angeles (UCLA) to teach Satellite Networks class. Now he teaches Satellite Telecommunications and Signals and Transmission.

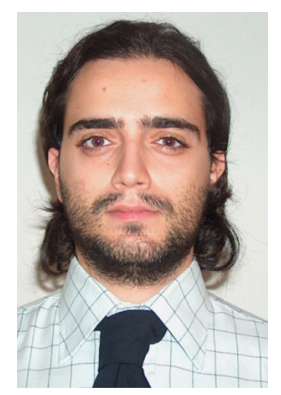

Cesare Roseti graduated cum laude in 2003 in Electronic Engineering at University of Rome 'Tor Vergata'. In 2003 and 2004 he was a visiting student at Computer Science Department of University of California, Los Angeles (UCLA). Since 2004 he is a $\mathrm{PhD}$ student at the Electronic Engineering Department. From August to December 2005 he worked at the TEC-SWS division of the European Space Agency (Noordwijk, The Netherlands). His research interests include satellites communications and protocol design, implementation and performance analysis in wired/ wireless networks. 